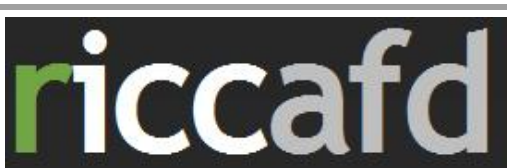

Revista Iberoamericana de Ciencias de la Actividad Física y el Deporte

\title{
PERFIL ANTROPOMÉTRICO DE JUDOCAS DE ÉLITE Y JUVENILES EN LA MODALIDAD DE COMBATE
}

\section{JUDOKAS ANTHROPOMETRIC PROFILE OF ELITE AND YOUTH IN COMBAT MODE}

\section{Rodríguez, G.A}

Docente Investigador

Universidad Sonto Tomas de Aquino.

Profesional en Cultura Física, Deporte y Recreación, Universidad Santo Tomas-Seccional Bucaramanga. (Col). Mágister en teoría y metodología del entrenamiento deportivo para la alta competencia. Instituto de Cultura Física Manuel Fajardo, Universidad del Deporte Cubano. Habana-Cuba. Email: guillermoarg0701@hotmail.com

\section{Código UNESCO: 5802.99}

Clasificación Consejo de Europa: 5

Recibido el 7 de junio de 2013

Aceptado el 1 de julio de 2013

PALABRAS
CLAVE:
Judo,
Deportistas
Elites y
Juveniles,
Masa magra,
Masa magra,
Composición
corporal,
Somatotipo.

KEY WORDS: Judo Elite Athletes and Young, Lean mass, Lean mass, Body composition, Somatotype.

\section{RESUMEN}

El tipo de estudio de esta investigación observacional descriptivo de corte transversal cuyo objetivo fué determinar y analizar el perfil antropométrico de los judocas santandereanos elites y juveniles en la modalidad de combate. El porcentaje de masa ósea y porcentaje de masa residual, de los judocas elites es $15,41 \%, 24.1 \%$, mientras que los judocas juveniles presentan porcentaje de $18,021 \%, 24.1 \%$ respectivamente.

Para las judocas elites y juveniles la masa ósea y porcentaje de masa residual media $15,4 \%$, $20,91 \%$ y 14,19 y 20,9 respectivamente. El componente masa grasa la media es de $13,17 \%$ de acuerdo a la clasificación ofrecida por Yuhasz la muestra de estudio se clasifica en percentil $P>91$ que se describe como muy alto. En la categoría juvenil el componente masa magra es de 7,36 el cual se encuentra en un percentil P21-40 que se describe como adecuado, las judocas elites la masa grasa es de $20,68 \%$, clasificada en percentil $P>91$ que se describe como muy alto, en la cual de acuerdo a la desviación estándar 5,25 la muestra no presenta homogeneidad., en la categoría juvenil el porcentaje del componente masa magra la media es de 27,26 , en el percentil $P>91$ que se describe como muy alto.La masa muscular de los judocas elites es de un $46.72 \%$, en cuanto a las judocas elites, los valores de la composición corporal son de un $43.01 \%$; y las judocas juveniles la media es de $37.64 \%$, mostrando una diferencia significativa con los judocas elites.

\section{ABSTRACT}

The type of observational research study of this cross-sectional descriptive aim was to identify and analyze the anthropometric profile of elite and youth judo Santander in combat mode. The percentage of bone and residual mass percentage of the elite judo is $15.41 \%, 24.1 \%$, while the percentage of judo presented 18,021 juveniles $\%$, 24.1\% respectively. For judo elite and juvenile bone mass and average residual mass percentage $15.4 \%, 20.91 \%$ and 14.19 and 20.9 respectively. The average fat mass component is $13.17 \%$ according to the classification given by Yuhasz the study sample is classified into percentile $P>91$ which is described as very high. At youth lean component is 7.36 which is in a percentile P21-40 is described as suitable, the elite judo fat mass is $20.68 \%$, rated percentile $P>91$ which described as very high, which according to the standard deviation 5.25 presents the sample homogeneity., in the junior category the percentage of lean mass component, the average is 27.26 , in percentile $P>91$ which very alto.La described as the muscle is an elite judo $46.72 \%$, in terms of judo elite values of body composition are of $43.01 \%$ and juveniles judo average is $37.64 \%$, showing a significant difference with elite judokas. 


\section{INTRODUCCIÓN}

Esta Los estudios sobre el crecimiento y desarrollo, con perspectiva antropológica, realizados en la población extranjera son cada vez más numerosos, a comparación de nuestro país que los trabajos que versan sobre la dinámica del somatotipo del judoca son más escasos. En el exterior los trabajos cada día se centran más en deportistas de competición.

Las investigaciones llevadas a cabo en esta población, son muy escasas no obstante se observa en los últimos años un creciente interés por incorporar nuevas metodologías para la evaluación del tamaño y la forma y composición corporal de los individuos, que incluyen el cálculo del somatotipo.

La antropometría básica nos puede mostrar información bastante importante en cuanto a las dimensiones corporales en los deportista elites y juveniles. La determinación de la estructura corporal a partir de algunas variable antropométricas desde hace ya algún tiempo viene siendo un factor primordial en la formación integral de los atletas y en especial en deportes como el judo donde el composición corporal es de gran incidencia en el resultado deportivo. Así mismo constituye un elemento bastante importante en la detección y selección de talentos deportivos. Es por esto que tener una adecuada composición morfológica ayuda al rendimiento deportivo.
Las variables morfológicas nos permiten en su valoración determinar la composición corporal y el somatotipo de los atletas. Heath y Carter 1967, y las mismas definen el somatotipo como la conformación morfológica presente. Por ende el somatotipo permite observar la forma del físico corporal en 3 dimensiones: Ectomorfia, Mesomorfia y Endomorfia (Norton y cols, 1996).

Es por esto que debemos entender que el somatotipo tiene gran relación con otras capacidades físicas. Cada disciplina deportiva exige diferentes tipos de somatotipo, sin embargo cada deporte debe tener unas capacidades específicas, pero debemos tener en cuenta que no solo con la técnica y el entrenamiento se pueden tener resultados de rendimiento y describir al deportista en su totalidad. (Heath y carter, 1990).

El estudio de la composición corporal permite evaluar a los deportistas en cuanto a su peso corporal, si se encuentra en optimas condiciones 0 por el contrario están desnutridos o con sobrepeso, de tal manera que estos datos ayuden a proveer guías para un adecuado manejo nutricional así como también permita evaluar su composición corporal y clasificarlos según su somatotipo, el cual es de vital importancia para el seguimiento y evolución del somatotipo en el tiempo, como también brindará herramientas necesarias para identificar talentos deportivos en procesos de formación.

Rev. Ib. CC. Act.Fis. Dep. 
Como todas las disciplinas de combate el judo debe tener en cuenta el control de su peso corporal para poder proyectar a sus rivales a la hora de competir y además tener un biotipo que constituya el rendimiento deportivo óptimo en el judoca santandereano. Esta valoración adquiere una mayor importancia cuando el peso del cuerpo debe trasladarse contra la gravedad, pasando a formar parte de una de las variables determinantes del rendimiento deportivo.

Es importante analizar los cambios del somatotipo de acuerdo al momento que se encuentra la preparación y a la categoría de peso a la que pertenezca, de manera que se pueda establecer la relación óptima del mismo con el rendimiento deportivo. (Carratalá, Benavent, Carqués, 2004).

La poca información acerca de las características antropométricas de los judocas, no permite conocer cómo evolucionan morfológicamente durante un plan de entrenamiento, por esta razón se hace necesario determinar el perfil antropométrico que permita obtener patrones que indiquen las características en cuanto a su composición corporal y de esta manera mejorar los procesos de preparación del judoca.

\section{MATERIAL Y MÉTODO}

La investigación se basa en un estudio descriptivo - explicativo ya que se describieron las manifestaciones de acuerdo a las pruebas aplicadas a la muestra. Es de tipo transversal por que la información se recolecto en un sólo momento.

Para la muestra se destinaron todos los integrantes de la selección Santander de judo de las categorías elite y juvenil, la cual corresponde a un total de 20 deportistas distribuidos así: 7 deportistas elite y 13 deportistas juveniles. El motivo de realizar la medición a todos los deportistas es minimizar los sesgos de selección. El estudio se realizó en los laboratorios del INDERSANTANDER. Instituto departamental para la recreación y el deporte. La recolección de datos se tomó al inicio de la temporada el cual se estableció en común acuerdo con el entrenador principal y el presidente de liga.

Los atletas se clasificaron en dos grupos elites y juveniles de acuerdo a la edad, experiencia deportiva y nivel de rendimiento. Teniendo esto en cuenta se tomaron las siguientes variables. Porcentaje de grasa, peso muscular, óseo, residual y graso, componentes Endomorficos, Ectomorficos, Mesomorficos, talla, peso, edad, sexo.

Todas las mediciones antropométricas se realizaron con el manual de lineamiento de antropometría Norton y Cols, (1996), también se calculo el error técnico de medición, que define como la desviación estándar de medidas repetidas tomadas individualmente una de otra en el mismo sujeto

Rev. Ib. CC. Act.Fis. Dep. 
Para la cuantificación de la composición corporal se aplicó el protocolo utilizado por el grupo español de cineantropometría fundamentados en la propuesta de De Rose y Guimaraes, en el cual se tienen en cuenta cuatro componentes corporales: masa ósea, masa residual, masa grasa y masa muscular. Acero (2002: 120).

Para el cálculo del somatotitpo se emplearon las ecuaciones de descritas por (Heath Carter, 1990), en cuanto a la composición corporal se realizo Formula de Von Doblen (1964) y modificada por Rocha (1975) para el análisis de la masa ósea, yuhasz, 1974, para el porcentaje de grasa y la formula de Rose y Guimares 1980, 1984, para la masa muscular.

Se realizo estadística descriptiva para determinar medidas de tendencia central (Media) y medidas de dispersión (Desviación estándar), mínimos y máximos de las características de los resultados de los judocas, con el propósito de establecer diferencias por cada categoría de peso como de nivel a partir de Microsoft Excel.

Para el desarrollo de la investigación se aplicaron métodos de nivel teóricos, empíricos y matemáticos-estadísticos:

\section{Métodos Teóricos:}

Método analítico-sintético: Para penetrar en la esencia del objeto de estudio y sistematizar la información necesaria para la determinar el perfil de cada judoca.

Histórico-lógico: para poder fundamentar el estudio, desde el punto de vista teórico, analizar los resultados y llegar a conclusiones.

Inducción-deducción: para llevar el análisis de valoración de los resultados en base a la a cada categoría que pertenece en su desempeño.

Sobre la base de la revisión de todas las disposiciones oficiales que norman el trabajo y atendiendo a las exigencias de la labor diagnóstico investigativa acometida en el presente trabajo, se emplearon los siguientes métodos empíricos:

Método de análisis de documentos: Para lograr una adecuada comprensión de las orientaciones y disposiciones del departamento metodológico de INDERSANTANDER, en cuanto a la preparación de los judocas en lo correspondiente a los componentes de la preparación en cada sesión de entrenamiento.

Método de la observación: Permitió constatar en la práctica los componentes antropométricos.

Matemáticos-estadísticos: método de la estadística descriptiva, el análisis porcentual, la media, desviación estándar.

\section{RESULTADOS}

En la tabla 1 nos muestra las características generales de la muestra de estudio distribuidos en grupos según su categoría y genero

Rev. Ib. CC. Act.Fis. Dep. 
observándose diferencias significativas en los judocas elites y juveniles en la variable peso corporal.

TABLA 1. Características generales por categorías y géneros.

\begin{tabular}{|c|c|c|c|c|c|c|}
\hline \multirow[b]{2}{*}{ Categoría } & \multirow[b]{2}{*}{ Genero } & \multirow[b]{2}{*}{ Estadística } & \multirow[b]{2}{*}{ Edad } & \multicolumn{2}{|c|}{ Talla } & \multirow{2}{*}{$\begin{array}{c}\text { Masa corporal } \\
(\mathrm{Kg})\end{array}$} \\
\hline & & & & $\mathbf{c m}$ & $\mathbf{m}$ & \\
\hline \multirow{4}{*}{ Elite } & \multirow{2}{*}{ Masculino } & Media & 22.17 & 177.1 & 1.77 & 87.17 \\
\hline & & Desvts & 8.26 & 9.22 & 0.092 & 27.61 \\
\hline & \multirow{2}{*}{ Femenino } & Media & 26 & 157 & 1.57 & 57.2 \\
\hline & & Desvts & 0 & 7.07 & 0.007 & 1.13 \\
\hline \multirow{4}{*}{ Juvenil } & \multirow{2}{*}{ Masculino } & Media & 14.83 & 172.3 & 1.72 & 67.9 \\
\hline & & Desvts & 1.16 & 4.179 & 0.048 & 17.88 \\
\hline & \multirow{2}{*}{ Femenino } & Media & 17 & 153.33 & 1.533 & 56.8 \\
\hline & & Desvts & 0 & 2.88 & 0.02 & 1.9 \\
\hline
\end{tabular}

En la tabla 2. Se describen las características del somatotipos de los judocas elites y juveniles en la rama masculina evaluados en cada categoría. De acuerdo con la perspectiva de los resultados de la media y la desviación estándar independientemente de la categoría nos podemos dar cuenta que el componente meso mórfico es dominante.

TABLA 2. Somatotipo promedio y desviación estándar en la rama masculina

\begin{tabular}{lcccc}
\hline & \multicolumn{2}{c}{ Elites } & \multicolumn{2}{c}{ Juveniles } \\
& Media & Desvt & Media & Desvt \\
\cline { 2 - 5 } Endomorfia & 4.57 & 2.49 & 3.41 & 4.28 \\
Mesomorfia & 6.16 & 1.38 & 1.29 & 0.94 \\
Ectomorfia & 1.5 & 1.05 & 2.77 & 1.54 \\
Somatotipo & Mesomorfico-Endomorfico & Mesomorfico-Endomorfico \\
\hline
\end{tabular}

En el Gráfico 1 se muestra el somatotitpo. Los componentes fueron heterogéneos en la muestra masculina.

Grafico 1. Representación grafica del somatotipo en función de las categorías elites y juveniles en la rama masculina.

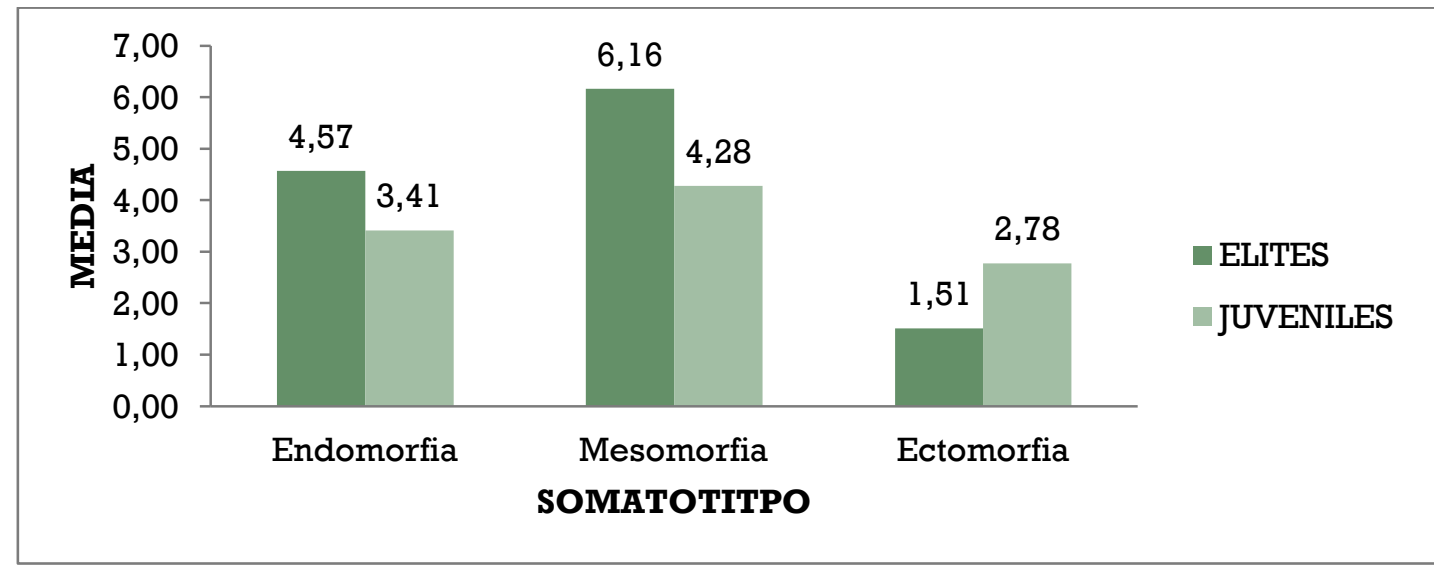

Rev. Ib. CC. Act.Fis. Dep. 
En la tabla 3. Se describen las características del somatotipos de las judocas elites y juveniles en la rama femenina evaluadas en cada categoría. De acuerdo con la perspectiva de los resultados de la media y la desviación estándar independientemente de la categoría nos podemos dar cuenta que el componente Mesomorfico dominante.

TABLA 3. Somatotipo promedio y desviación estándar en la rama femenina.

\begin{tabular}{lcccc}
\hline & \multicolumn{2}{c}{ Elites } & \multicolumn{2}{c}{ Juveniles } \\
& Media & Desvt & Media & Desvt \\
\cline { 2 - 5 } Endomorfia & 5.8 & 1.52 & 8.29 & 0.35 \\
Mesomorfia & 5.01 & 0.69 & 4.99 & 0.66 \\
Ectomorfia & 1.42 & 0.95 & 0.86 & 0.55 \\
Somatotipo & Endomorfico-Mesomorfico & Endomorfico-Mesomorfico \\
\hline
\end{tabular}

En el Gráfico 2 se muestra el somatotipo. Los componentes fueron heterogéneos en la muestra femenina.

Gráfico 2. Representación grafica del somatotipo en función de las categorías elites y juveniles en la rama femenina.

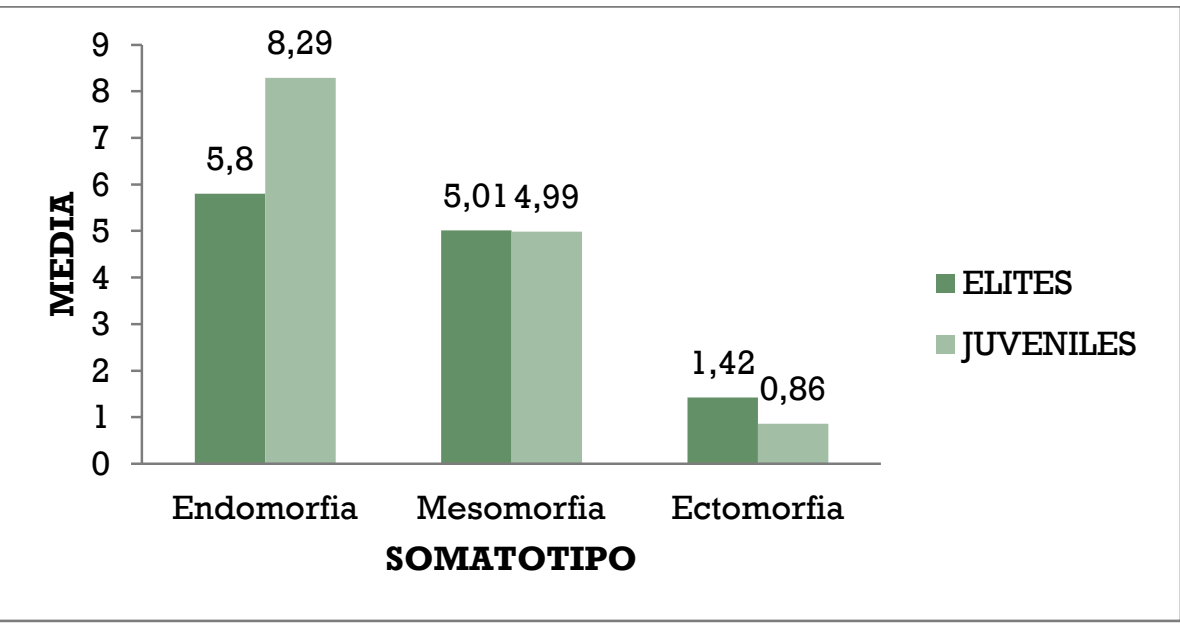

Tabla 4. Se describen las características de la composición corporal de los judocas elites y juveniles en la rama masculina evaluados en cada categoría. De a cuerdo con la perspectiva de los resultados de la media y la desviación estándar se manifiesta diferencias significativas en la variables masa ósea. masa grasa. masa libre en grasa.

Rev. Ib. CC. Act.Fis. Dep. 
TABLA 4. Composición corporal. promedio y desviación estándar en la rama masculina.

\begin{tabular}{lcccccccc}
\hline & \multicolumn{4}{c}{ Elites } & \multicolumn{4}{c}{ Juveniles } \\
\cline { 2 - 10 } & \multicolumn{2}{c}{ Media } & \multicolumn{2}{c}{ Desvt } & \multicolumn{2}{c}{ Media } & \multicolumn{2}{c}{ Desvt } \\
Masa Ósea & $\mathbf{K g}$ & $\%$ & $\mathbf{K g}$ & $\%$ & $\mathbf{K g}$ & $\%$ & $\mathbf{K g}$ & $\%$ \\
\cline { 2 - 10 } Masa Residual & 13 & 15.41 & 2.45 & 2.14 & 11.9 & 18.02 & 1.3 & 2.89 \\
Masa Grasa & 21 & 24.1 & 6.65 & 0 & 16.4 & 24.1 & 4.3 & 0 \\
Masa Muscular & 13.91 & 13.76 & 14.2 & 8.81 & 7.36 & 19.01 & 4.9 & 3.55 \\
Masa libre en Grasa & 39.24 & 46.72 & 6.43 & 6.72 & 32.3 & 47.77 & 7.9 & 2.31 \\
\hline
\end{tabular}

En el Gráfico 3 se muestra la composición corporal de los judocas de la rama masculina.

Gráfico 3. Representación grafica de la composición corporal de las categorías elites y juveniles en la rama masculina.

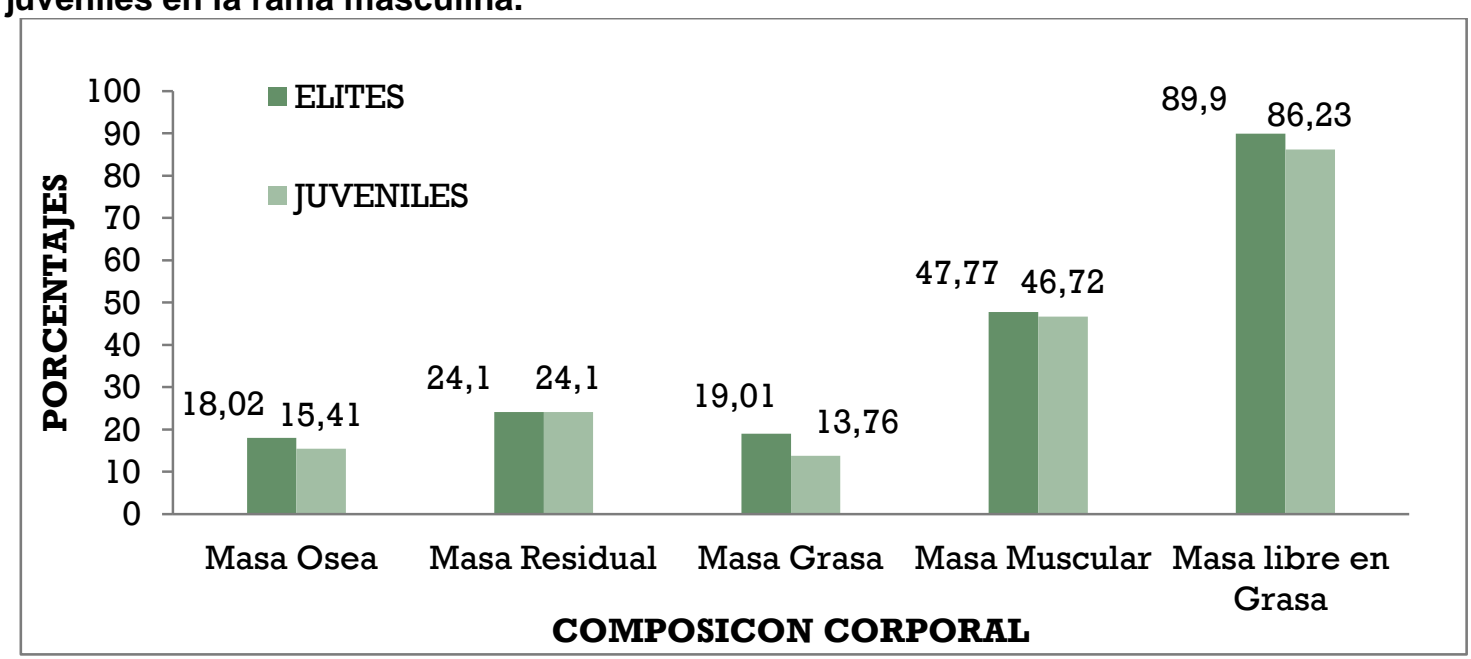

Gráfico 4. Análisis de la composición corporal en la variable masa grasa en la categoría elite y juvenil. Atendiendo al análisis entre estas categorías se puso de manifiesto que el porcentaje de tejido adiposo es de $13.17 \%$ de acuerdo a la clasificación ofrecida por Yuhasz. (Citado por Acero: 2000: 112) nos muestra de estudio se clasifica en percentil $P>91$ que se describe como muy alto. en esta categoría elite. Mientras que en la categoría juvenil el porcentaje del el tejido adiposo es de de 7.36 de acuerdo a clasificación estaríamos hablando que esta muestra de estudio está en el percentil P21-40 que se describe como adecuado. La evaluación de dicho componente corporal pro cada categoría nos mostro mayores porcentaje de tejido adiposo subcutáneo en la categoría elite.

Rev. Ib. CC. Act.Fis. Dep. 
La Grafica 4. Componente graso (porcentaje sobre la masa corporal) de los judocas de la rama masculina.

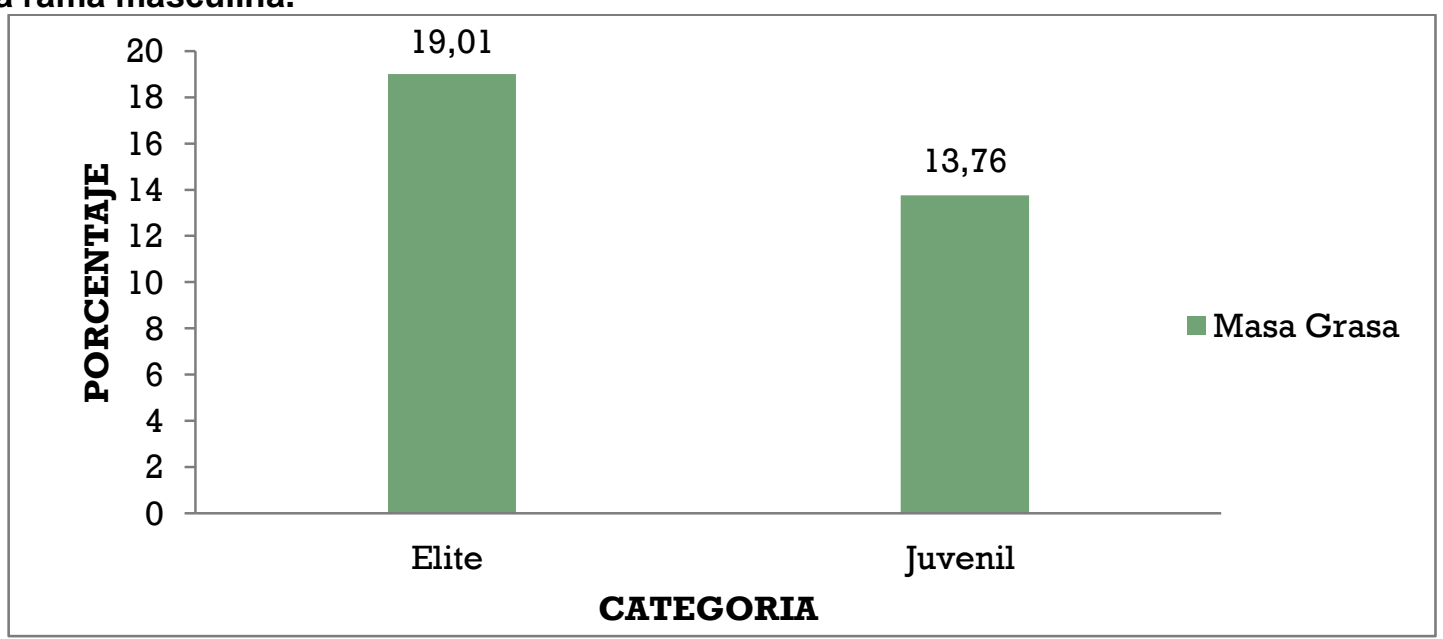

Tabla 5. Se describen las características de la composición corporal de las judocas elites y juveniles en la rama femenina evaluados en cada categoría. De a cuerdo con la perspectiva de los resultados de la media y la desviación estándar se manifiesta diferencias significativas en la variables masa ósea, masa grasa, masa muscular y masa libre en grasa.

TABLA 5. Composición corporal. promedio y desviación estándar en la rama femenina

\begin{tabular}{lccccccccc}
\hline & \multicolumn{4}{c}{ Elites } & \multicolumn{4}{c}{ Juveniles } \\
\cline { 2 - 10 } & \multicolumn{2}{c}{ Media } & \multicolumn{2}{c}{ Desvt } & \multicolumn{2}{c}{ Media } & \multicolumn{2}{c}{ Desvt } \\
Masa Ósea & $\mathbf{K g}$ & $\%$ & $\mathbf{K g}$ & $\%$ & $\mathbf{K g}$ & $\%$ & $\mathbf{K g}$ & $\%$ \\
\cline { 2 - 10 } Masa Residual & 8.85 & 15.47 & 0.55 & 0.66 & 8.04 & 14.19 & 0.45 & 1.25 \\
Masa Grasa & 11.87 & 20.9 & 0.23 & 0 & 11.87 & 20.9 & 15.47 & 27.26 \\
Masa Muscular & 11.75 & 20.6 & 2.77 & 5.25 & 15.47 & 27.26 & 0.1 & 1.07 \\
Masa libre en Grasa & 24.63 & 43.01 & 3.1 & 4.58 & 21.5 & 37.64 & 2.06 & 2.32 \\
\hline
\end{tabular}

En el Gráfico 5 se muestra la composición corporal de los judocas de la rama femenina.

Rev. Ib. CC. Act.Fis. Dep. 
Gráfico 5. Representación grafica de la composición corporal de las categorías elites y juveniles en la rama femenina

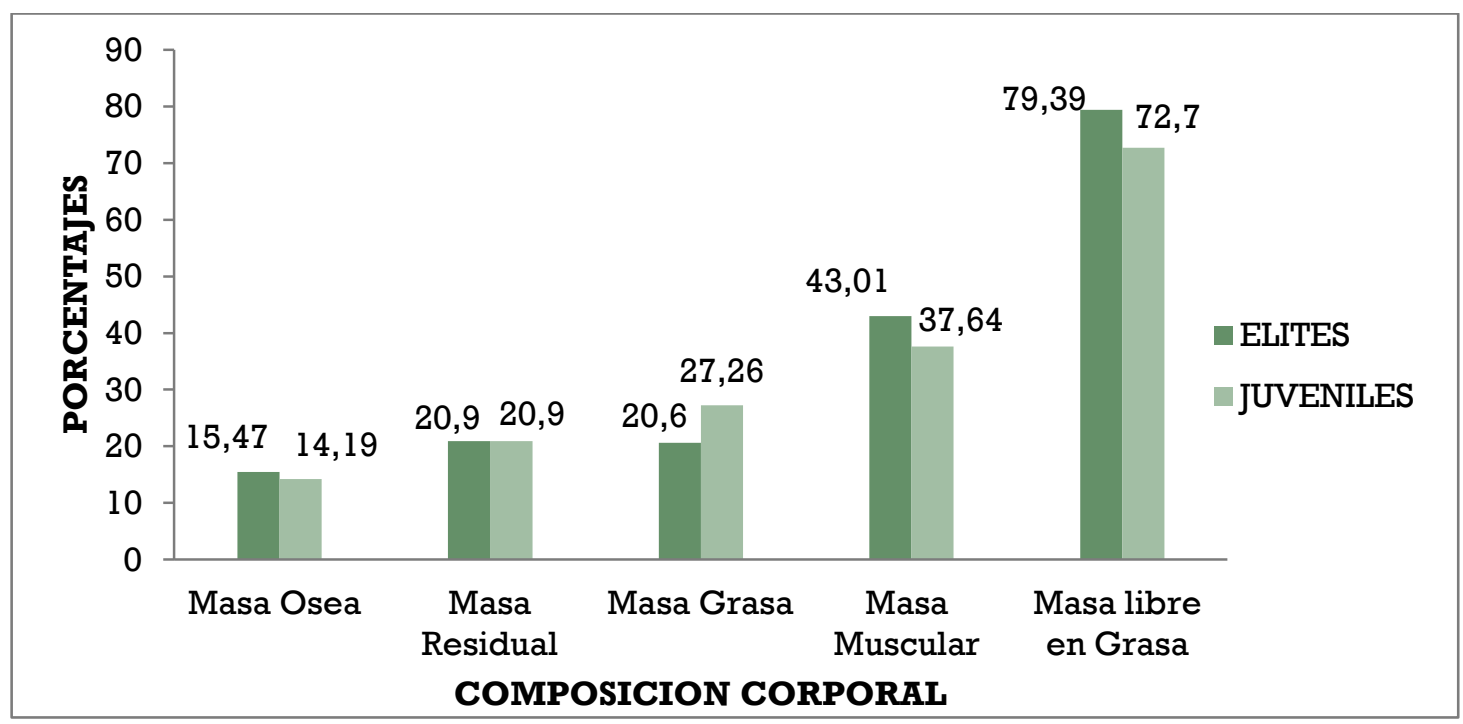

Gráfico 6. Análisis de la composición corporal en la variable masa grasa en la categoría elite y juvenil. Atendiendo el análisis entre las categorías se puso de manifiesto que el porcentaje de tejido adiposo es de $20.68 \%$ de acuerdo a la clasificación ofrecida por Yuhasz. (Citado por Acero: 2000: 112) la muestra de estudio se clasifica en percentil $P>91$ que se describe como muy alto, en la categoría elite. Mientras que en la categoría juvenil el porcentaje del el tejido adiposo es de 27.26 de acuerdo a clasificación estaríamos hablando que esta muestra de estudio está en el percentil $P>91$ que se describe como muy alto. La evaluación de dicho componente corporal por cada categoría nos mostró mayores porcentaje de tejido adiposo subcutáneo en la categoría juvenil

Gráfico 6. Componente graso (porcentaje sobre la masa corporal) de las judocas de la rama femenino

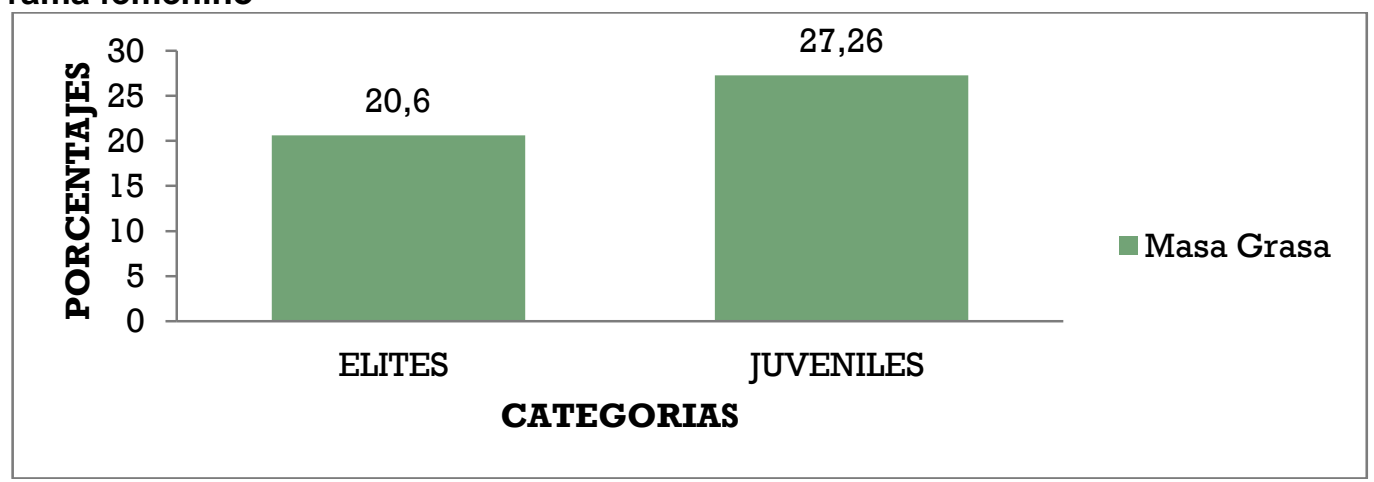

\section{DISCUSIÓN}

Es importante mencionar que los deportistas fueron separados. en elites y juveniles a partir de su edad y nivel competitivo. en Rev. lb. CL. Act.Fis. Dep. hombres y mujeres. También es pertinente apuntar que el grupo de deportistas en la rama femenina es poco representativo debido a la baja participación de mujeres en la 
selección Santander de judo y por ende la muestra es mínima. Es muy importante saber que los datos antropométricos brindados en este estudio nos permiten observar el grado nutricional que tienen los judocas a través de la composición corporal determinada en la investigación (Sarria A. Moreno I. Bueno M.1985)

En términos generales podríamos decir que la masa muscular a medida que va avanzando la edad aumenta de manera gradual con respecto a la ganancia de peso corporal del individuo. Gran parte de este incremento se debe a la relación de testosterona que se da en estas edades. Hecho que concuerda con el mayor porcentaje de masa muscular evidenciada en la categoría elite de la muestra de estudio en la rama masculina. Este hecho es de gran pertinencia a la hora de poder crear modelos de entrenamiento, llevándonos a potenciar los trabajos de fuerza muscular y por ende el rendimiento deportivo. Todo supeditado a que a mayor adiposidad corporal. se tiene la tendencia a disminuir o influir negativamente en el rendimiento deportivo de este tipo de deporte donde la composición corporal es de vital importancia a la hora de ganar un combate.

Todo fundamentado en que al aumentar el peso corporal y el mismo no es articulado y acompañado de un proceso para producir más fuerza. Si miramos desde el punto de vista que el judo es una disciplina deportiva que necesita mayor aceleración para proyectar a su contrincante y la misma es directamente proporcional a la fuerza pero inversamente proporcional a la masa corporal, entonces estaríamos hablando que el exceso de grasa a un nivel de esfuerzo dado en la aplicación de la fuerza va influir negativamente en los movimiento veloces y potentes que tiene que hacer el judoca a la hora de intentar proyectar a su rival, además que este aumento de adiposidad incremente el costo metabólico a la hora de realizar la actividad física ya que hay que mover mayor masa corporal.

Por último es de resaltar que todos los deportes que implique mover su masa corporal en contra de la gravedad se beneficiaran de un porcentaje de grasa bajo, como lo muestra este estudio en los deportistas elites de la rama masculina.

Donde hay una marcada tendencia a la endomorfia es en la rama femenina, donde esta diferencia en la forma física puede estar enmarcada por el tipo de entrenamiento que lleva una y otra categoría a la hora de aplicar las cargas de entrenamiento. Es importante tener en cuenta que la composición corporal de las judocas femeninas en las dos categorías este enmarcada por la endomorfia podríamos decir que este aspecto podría ser explicado por el reducido tamaño de la muestra de estudiada.

Los judocas tienen una difícil tarea de mantener un equilibrio en el consumo y gasto de energía de tal manera que les permite tener un

Rev. Ib. CC. Act.Fis. Dep. 
mejor rendimiento en los entrenamientos como en las competencias nacionales e internacionales. lo cual es una tarea difícil por las altas demandas de energía que se requieren son grandes.

\section{CONCLUSIONES}

El somatotipo medio varia en las dos muestras en función de la edad $y$ el nivel competitivo. La mesomorfía es la variable que más influye en la rama masculina en sus dos categorías. mientras que la endomorfia predomina en la rama femenina con un porcentaje bastante alto y marcado en la categoría juvenil.

En la variación de la fisonomía en el sexo de los individuos estudiados encontramos diferencias. Dentro de los tres componentes la endomorfia nos contribuye a marcar la diferencia en las dos ramas. De tal manera que los judocas elites y juvenil hombres tiene somatotipo mesomorfico endomorfico mientras que las mujeres en estas dos categorías predomina la endomorfia mesomorfia.

En cuanto a los valores de la composición corporal nos muestra que los judocas juveniles poseen mayor porcentaje de masa muscular y masas libre en grasa que los judocas elites. Mientras que en la rama femenina sucede totalmente lo contrario las judocas elites tienen mejor porcentaje de masa muscular y masa libre de grasa que las judocas juveniles.

\section{Recomendaciones}

Los resultados que se encontraron nos pueden indicar que se necesita profundizar y poner especial atención al control antropométrico de cada uno de los deportistas en su respectiva categoría de peso con el fin de optimizar el rendimiento deportivo de los judocas.

Desde la metodología del entrenamiento se recomienda ampliar la muestra para futuras investigaciones para aumentar la confiablidad de los resultados especialmente en la rama femenina.

Perfeccionar los planes de entrenamiento de los judocas santandereanos en relación al control de la composición corporal (antropometría) con el fin de mejorar el rendimiento deportivo en competencias nacionales e internacionales.

\section{REFERENCIAS BIBLIOGRÁFICAS}

1. Acero. J. Cineantropometría. Fundamentos y procesos. Cali: Faid. 2002.

2. Carratalá. V. Benavent. J; Carqués. L. 2004. UIRFIDE. Departamento de Educación Física y Deportiva. Universitat de Valencia. III Congreso de la Asociación Española de Ciencias del Deporte: "Hacia la convergencia Europea. Valencia.

3. Carter. J.E.L.; and Heath. B. (1990). Somatotyping Development and Applications. Cambridge. Cambridge University Press.

4. Norton $\mathrm{K}$ and Olds $\mathrm{T}$. Antropométrica Sydney -Australia: UNSW PRESS. 1996.

5. Sarria A. Moreno L. Bueno M: Métodos de valoración de la maduración ósea. Rev Esp Pediatr 1985; 41: 91

6. Ceballos J; González P. Manual De Antropometría. Habana. Cuba. 2003.

Rev. lb. Cᄃ. Act.Fis. Dep. 
7. Burrows A. Díaz N. Muzzo. S. (2004) Variaciones del índice de masa corporal (IMC) de acuerdo al grado de desarrollo puberal alcanzado. Rev Med. 2004

8. Campbell. D.T. \& Stanley. J.C. (1966). Experimental and Quasi-Experimental Designs for Research. Skokie. IL: Rand McNally.

9. Jackson A. Pollock ML y WARD A Generalized Equations for predicting body density of woman. Med Sci sport Exerc 1980. 12: 175-182.

10. Marfell. Jones. M. Kinanthreport 2003. ISAK.

11. Norton K. Tim Olds. ANTROPOMETRICA (2000). Edición en Español: Dr. Juan Carlos Mazza. Biosystem Sevicio Educativo

Rev. Ib. Cᄃ. Act.Fis. Dep. 